\title{
Origin of the response of nanomechanical resonators to bacteria adsorption
}

\author{
D. Ramos, J. Tamayo, ${ }^{\text {a) }}$ J. Mertens, and M. Calleja \\ BioNanoMechanics Laboratory, National Centre for Microelectronics, IMM-CNM, CSIC Isaac Newton 8 \\ (PTM), Tres Cantos, Madrid 28760, Spain

\begin{abstract}
A. Zaballos
Genomics Functional Unit, Department of Immunology and Oncology, National Center for Biotechnology, CSIC, Campus de Cantoblanco, Darwin 3, Madrid 28049, Spain
\end{abstract}

(Received 5 June 2006; accepted 31 August 2006; published online 30 November 2006)

\begin{abstract}
Resonant microcantilevers are being actively investigated as sensitive mass sensors for biological detection. By performing experiments of adsorption of the bacteria Escherichia coli on singly clamped microcantilevers, we demonstrate that the effect of the added mass is not the only and may not be the main origin of the response of these sensors. The experiments show that the magnitude and sign of resonance frequency shift both depend critically on the distribution of the adsorbed bacterial cells on the cantilever. We relate this behavior to the added mass that shifts the resonance to lower frequencies and the higher effective flexural rigidity of the cantilever due to the bacteria stiffness that shifts the resonance to higher frequencies. Both effects can be uncoupled by positioning the cells where each effect dominates, near the free cantilever end for measuring the added mass or near the clamping for measuring the increase of flexural rigidity. () 2006 American Institute of Physics. [DOI: 10.1063/1.2370507]
\end{abstract}

There is a need for biochemical sensors capable of improving the sensitivity, throughput, and rapidity of the assays for pharmaceutical and biological research as well as for medical diagnostics. Nanomechanical resonators, such as singly and doubly clamped microcantilevers, are emerging as an attractive alternative approach to current technologies. ${ }^{1-9}$ They provide label-free detection and can simply be scaled down in size, correspondingly reducing the required sample volume, and scaled up in number of elements for multiple and complex biochemical analysis. ${ }^{2}$ The basic principle of biological sensors based on nanomechanical resonators is the measurement of the resonance frequency change due to the added mass of the biomolecules bound to the cantilever surface. The detection of bacteria, viruses, and long DNA molecules at the level of a single entity has been reported. ${ }^{6-9}$ Moreover, unprecedented mass sensitivity is expected by shrinking the mechanical structures to the nanoscale and by a major development of the nanofabrication techniques. ${ }^{1}$

Reported experiments of biological sensing using nanomechanical resonators consist of (i) dipping the cantilever in the molecule solution, (ii) drying the cantilever, and (iii) measuring the resonance frequency in air or vacuum environments. The reason for the performance of these experiments ex situ, in air, or vacuum is the low quality factor found for cantilevers immersed in liquid. ${ }^{10,11}$ The mass of the molecules attached to the cantilever has been usually quantified by using

\footnotetext{
${ }^{a)}$ Author to whom correspondence should be addressed; electronic mail: jtamayo@imm.cnm.csic.es
}

$$
\frac{\Delta \omega}{\omega_{0}}=-\frac{1}{2} \frac{\Delta m}{m},
$$

where $\omega_{0}$ is the angular resonance frequency, $\Delta \omega$ is the angular resonance frequency shift, $m$ is the cantilever mass, and $\Delta m$ is the adsorbed mass. Whereas Eq. (1) works reasonably well for added mass uniformly distributed along the cantilever, it has been demonstrated that for discrete masses, their positions on the cantilever largely affect the frequency shift. $^{12}$

In this work, we have studied the effect of the adsorption of the bacteria Escherichia coli (E. coli) on the resonance frequency of the fundamental flexural mode of a singly clamped microcantilever.

Firstly, we measured the resonance frequency shift of the first flexural mode resulting from dipping silicon cantilevers in an $E$. coli suspension with a concentration of about $10^{9}$ cells $/ \mathrm{ml}$. The cantilevers were $500 \mu \mathrm{m}$ long, $100 \mu \mathrm{m}$ wide, and $1 \mu \mathrm{m}$ thick. The histogram of 29 measurements is shown in Fig. 1. The mean frequency shift for the first resonant mode is $-1.0 \%$. However, the histogram exhibits a broad distribution, indicating large irreproducibility. More strikingly, the histogram encloses a $24 \%$ of cases in which the frequency increases instead of decreasing. This frequency shift cannot be explained in terms of adsorbed mass [see Eq. (1)]. We relate this unexpected behavior to the local changes of the cantilever flexural rigidity at the positions where bacteria are bound, as will be demonstrated below.

To understand this behavior, we deposited by the ink-jet technique $^{13} 0.5 \mathrm{nl}$ droplets of bacteria in ultrapure water at different positions along the cantilever. Figure 2 shows the optical micrograph of three cantilevers, in which bacteria have been deposited near the free end, on the middle, and 


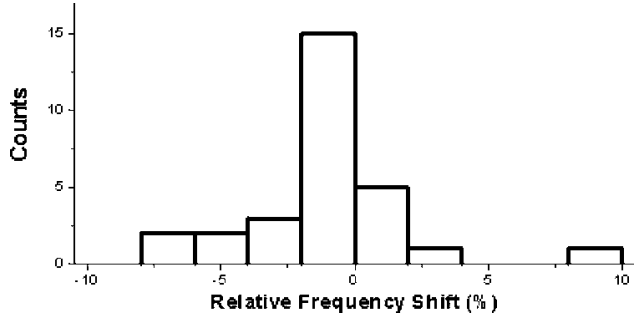

FIG. 1. Histogram of the frequency shift of the first resonant mode of a microcantilever in air due to the immersion of the microcantilever in an $E$. coli suspension with a concentration of about $10^{9}$ cells $/ \mathrm{ml}$. The cantilevers used are similar to those shown in Fig. 2.

near the clamping at three different positions. The bacteria adsorption follows a ringlike pattern that is reminiscent of the droplet shape. The number of E. coli cells deposited is between 3000 and 5000 .

Figure 3 shows the frequency spectra of the Brownian motion of the cantilevers shown in Fig. 2, before and after bacteria deposition. The fundamental resonance frequency before adsorption ranged from 7.0 to $8.5 \mathrm{kHz}$. For the cantilever in which bacteria were deposited near the free end, a negative resonance frequency shift of $-4.0 \%$ was observed, whereas for the $E$. coli cells bound on the cantilever middle region, the resonance frequency shift was negligible. More surprisingly, the resonance frequency increased by $3.6 \%$ when bacteria were deposited near the cantilever clamping. If we consider that the resonance frequency shift is due to the added mass, the resonance frequency shift should always be negative and its magnitude should vary from null for the case of bacteria positioned at the clamping to maximal for the case of bacteria adsorbed near the free cantilever end. ${ }^{12}$

Surface stress induced by molecular adsorption can have influence on the resonance frequency. ${ }^{11,14,15}$ However, this effect is not fully understood. Two theoretical models have been proposed. One simplifies the surface stress to an axial force exerted on the cantilever. ${ }^{16,17}$ It predicts changes in the resonance frequency per surface stress unit $(\mathrm{N} / \mathrm{m})$ of about $1 \%$ for cantilevers as those used here. We have measured the surface stress induced by bacteria adsorption by using a profiling technique recently developed. ${ }^{18}$ Our measurements indicate surface stress changes of $0.05-0.10 \mathrm{~N} / \mathrm{m}$, which are not sufficient to explain the obtained frequency shifts by ap-

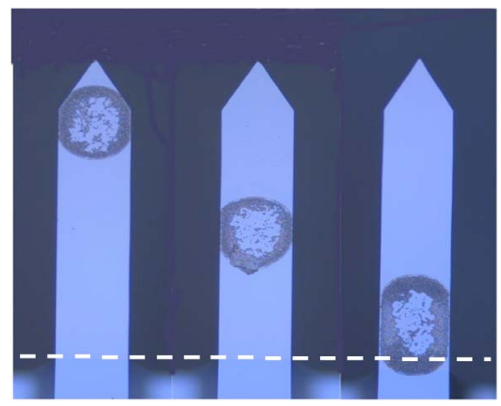

FIG. 2. Optical micrographs of three silicon cantilevers in which the bacterial cells have been deposited by ink jet at three different positions along the cantilever length. The cantilevers are $500 \mu \mathrm{m}$ long, $100 \mu \mathrm{m}$ wide, and $1 \mu \mathrm{m}$ thick. The center of the bacteria spot is separated from the clamping at 73,200 , and $390 \mu \mathrm{m}$, approximately. The dashed line indicates the clamping position.

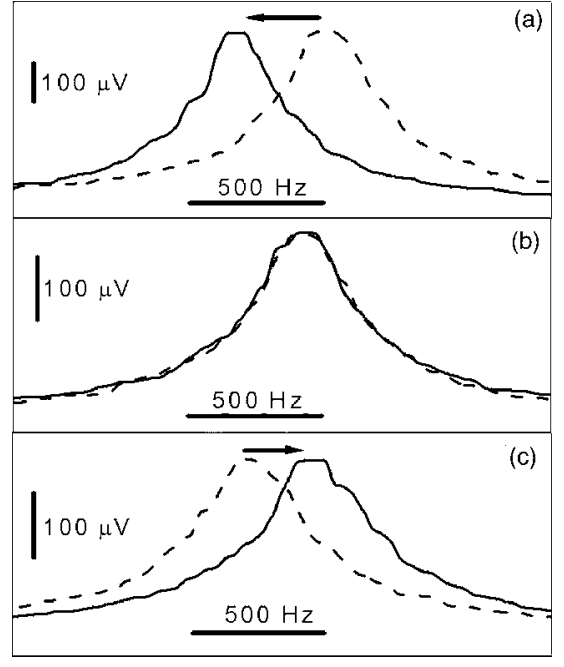

FIG. 3. Frequency spectra of the Brownian motion of the three cantilevers shown in Fig. 2, before (discontinuous line) and after (continuous line) bacteria deposition. The arrows indicate the sign of the resonance frequency shift. The measurements were performed in air. The shown frequency bandwidth allows the visualization of the first resonance mode located between 7.0 and $8.5 \mathrm{kHz}$. The frequency spectra results of averaging 50 curves. The center positions of the bacteria spot on the cantilevers with respect to the clamping are (a) $x_{0}=73 \mu \mathrm{m}$, (b) $x_{0}=200 \mu \mathrm{m}$, and (c) $x_{0}=390 \mu \mathrm{m}$.

plying this model. The second model states that the surface stress does not cause a resonance frequency shift as the axial force is relieved through the deformation of the free cantilever end. In this model, an induced strain-dependent surface stress is necessary to observe resonance changes. ${ }^{19}$ This effect produces changes of the resonant frequency smaller than $10 \mathrm{ppm}$, orders of magnitude smaller than the frequency shifts observed here.

To explain the results shown here, we propose a model that accounts for the mechanical properties of the attached bacteria that increase the stiffness of the cantilever. We model our cantilever as an Euler-Bernoulli beam, in which both the mass per unit length and the flexural rigidity are dependent on the longitudinal position. The differential equation of the vibration is given by

$$
(\rho S+\lambda(x)) \frac{\partial^{2} u(x, t)}{\partial t^{2}}+\frac{\partial^{2}}{\partial x^{2}}\left[D(x) \frac{\partial^{2} u(x, t)}{\partial x^{2}}\right]=0,
$$

where $u$ is the cantilever transverse displacement, $x$ is the longitudinal coordinate, $t$ is the time, $\rho$ is the cantilever mass density, $S$ is the cross-section area, $\lambda(x)$ is the adsorbed mass per unit length, and $D(x)$ is the flexural rigidity of the cantilever that is given by ${ }^{20}$

$D(x)=\frac{W}{12} \frac{E_{c}{ }^{2} T_{c}{ }^{4}+E_{b}{ }^{2} T_{b}{ }^{4}(x)+2 E_{c} E_{b} T_{c} T_{b}(x)\left[2 T_{c}{ }^{2}+2 T_{b}{ }^{2}(x)+3 T_{c} T_{b}(x)\right]}{E_{c} T_{c}+E_{b} T_{b}(x)}$,

where $W$ is the cantilever width, $T$ is the thickness, and $E$ is Young's modulus, whereas the subscripts $c$ and $b$ denote the cantilever and the bacteria, respectively. To calculate the resonance frequency we have applied Rayleigh's approximation. ${ }^{21}$ This method deduces the resonance frequencies by performing an energy-work balance and assuming that the eigenmode shapes are not substantially changed by the adsorbed bacteria. The average strain energy and kinetic energy 


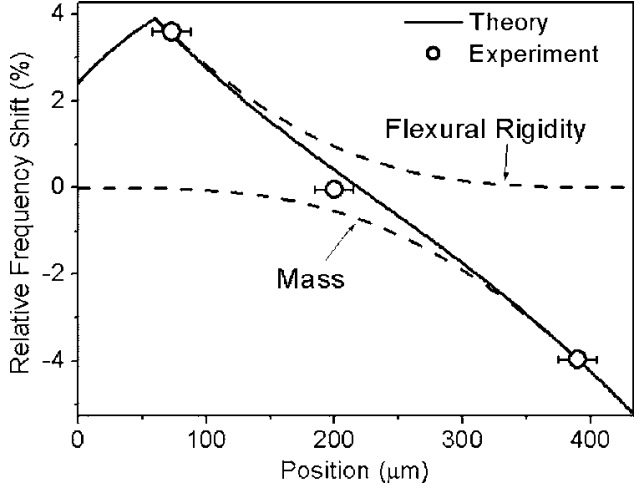

FIG. 4. Theoretical calculations of the resonance frequency shift as a function of the longitudinal position of the adsorbed bacteria with respect to the clamping. The dashed lines consider the separate effects of the increase of mass and flexural rigidity of the cantilever, whereas the solid line represents the resonance frequency shift considering both effects. The graph also includes the experimental data of the resonance frequency shift shown in Fig. 3 (circles). The error bar accounts for the uncertainty in the determination of the position of the adsorption center. This error is estimated to be $\pm 15 \mu \mathrm{m}$.

per oscillation cycle are, respectively, given by

$$
\begin{aligned}
& \left\langle W_{s}\right\rangle=\left\langle\frac{1}{2} \int_{0}^{L} D(x)\left(\frac{\partial^{2} u}{\partial x^{2}}\right)^{2} d x\right\rangle, \\
& \left\langle W_{k}\right\rangle=\left\langle\frac{1}{2} \int_{0}^{L}[\rho S+\lambda(x)]\left(\frac{\partial u}{\partial t}\right)^{2} d x\right\rangle,
\end{aligned}
$$

where $L$ is the cantilever length. By equating $\left\langle W_{k}\right\rangle$ and $\left\langle W_{s}\right\rangle$ and assuming a transverse vibration $u(x, t)=A \psi_{n}(x) \cos \left(\omega_{n} t\right.$ $+\phi)$, where $\psi_{n}$ is the eigenmode shape of the unloaded cantilever, ${ }^{1,21} \omega_{n}$ is the $n$th mode resonance frequency, and $A$ and $\phi$ are arbitrary values of the amplitude and phase of the vibration, the resonance frequency is calculated as

$$
\omega_{n}^{2}=\frac{\int_{0}^{L} D(x) \ddot{\psi}_{n}^{2}(x) d x}{\int_{0}^{L}[\rho S+\lambda(x)] \psi_{n}^{2}(x) d x} .
$$

We have applied Eq. (6) to estimate the first mode resonance frequency shift due to the increase of mass and flexural rigidity induced by the deposited bacteria. We model the adsorbed bacteria as a homogeneous and uniform disk with a diameter of $100 \mu \mathrm{m}$ that contains 4200 bacteria cells. The bacterium mass is $665 \mathrm{fg},{ }^{6}$ and the biolayer height is $840 \mathrm{~nm}$, smaller than the bacterium height of about $1 \mu \mathrm{m}$, to reflect that the adsorbed bacteria are not densely packed in the experiments. Young's modulus of $1.3 \mathrm{GPa}$ was chosen in order to mimic the experimental results. This value is similar to those obtained by atomic force microscopy measurements in dried bacteria. ${ }^{22}$ Figure 4 shows the calculated frequency shift versus the adsorption position along the cantilever due to the added mass (dashed line), the change of flexural rigidity (dashed line), and both effects (solid line). The experimental values of the resonance frequency shift obtained in Fig. 3 are also included (symbols). The theory shows a good agreement with the experimental data indicating the consistency of the presented model. The added mass of the adsorbed bacteria produces a negative resonance frequency shift whose magnitude varies from approximately null for the adsorption near the clamping to maximal for the adsorp- tion near the free cantilever end. Oppositely, the increased flexural rigidity due to the bacteria adsorption produces a positive resonance frequency shift whose magnitude varies from approximately null for the adsorption near the free cantilever end to maximal for the adsorption near the clamping. The resonance response can then be maximized by locating the adsorption on either near the clamping for measuring the positive resonance frequency shift induced by the bacteria stiffness or near the free end for measuring the negative frequency shift due to the adsorbed bacteria mass.

In conclusion, we have demonstrated that the response of nanomechanical resonators to bacteria adsorption does not only depend on the added mass, but also on the stiffness of the bacterial cells. Both effects can be uncoupled by positioning the bacterial cells where each effect dominates, near the free cantilever end for measuring the added mass or near the clamping for measuring the increase of flexural rigidity. Both geometries allow sensitive bacteria detection of about $0.1 \mathrm{~Hz}$ per bacterium. This sensitivity can be easily enhanced by at least one order of magnitude by using higher vibration modes or by scaling down the cantilever size. The results of this work can be generalized to the adsorption of any molecule. In addition, the effect of the mechanical properties of the adsorbed molecules will become increasingly important as the size of the resonators is decreased towards the nanoscale.

One of the authors (D.R.) acknowledges the fellowship funded by the Autonomic Community of Madrid. This work was supported by the Spanish National Research Council (CSIC), Project No. 200550M056.

${ }^{1}$ K. L. Ekinci and M. L. Roukes, Rev. Sci. Instrum. 76, 061101 (2005).

${ }^{2}$ N. V. Lavrik, M. J. Stepaniak, and P. G. Datskos, Rev. Sci. Instrum. 75, 2229 (2004).

${ }^{3}$ T. P. Burg and S. R. Manalis, Appl. Phys. Lett. 83, 2698 (2003).

${ }^{4}$ L. B. Sharos, A. Raman, S. Crittenden, and R. Reifenberger, Appl. Phys. Lett. 84, 4638 (2004).

${ }^{5}$ K. Y. Gfeller, N. Nugaeva, and M. Hegner, Biosens. Bioelectron. 21, 528 (2005).

${ }^{6}$ B. Illic, D. Czaplewski, H. G. Craighead, P. Neuzil, C. Campagnolo, and C. Batt, Appl. Phys. Lett. 77, 450 (2000).

${ }^{7}$ A. Gupta, D. Akin, and R. Bashir, Appl. Phys. Lett. 84, 1976 (2004).

${ }^{8}$ K. L. Ekinci, X. M. H. Huang, and M. L. Roukes, Appl. Phys. Lett. 84, 4469 (2004).

${ }^{9}$ B. Illic, Y. Yang, K. Aubin, R. Reichenbach, S. Krylov, and H. G. Craighead, Nano Lett. 5, 925 (2005).

${ }^{10}$ J. F. Vignola, J. A. Judge, J. Jarzynski, M. Zalautdinov, B. A. Houston, and J. W. Baldwind, Appl. Phys. Lett. 88, 041921 (2006).

${ }^{11}$ J. Tamayo, A. D. L. Humphries, A. R. Malloy, and M. J. Miles, Ultramicroscopy 86, 167 (2001).

${ }^{12}$ S. Dohn, R. Sandberg, W. Svendsen, and A. Boisen, Appl. Phys. Lett. 86, 233501 (2005).

${ }^{13}$ A. Bietsch, J. Zhang, M. Hegner, H. P. Lang, and Ch. Gerber, Nanotechnology 15, 873 (2004).

${ }^{14}$ J. H. Lee, T. S. Kim, and K. H. Yoon, Appl. Phys. Lett. 84, 3187 (2004).

${ }^{15}$ T. Ono and M. Esashi, Rev. Sci. Instrum. 76, 093107 (2005).

${ }^{16}$ G. Y. Chen, T. Thundat, E. A. Wachter, and R. J. Warmack, J. Appl. Phys. 77, 3618 (1995).

${ }^{17}$ A. W. Farland, M. A. Poggi, M. J. Doyle, L. A. Bottomley, and J. S. Colton, Appl. Phys. Lett. 87, 053505 (2005).

${ }^{18}$ J. Mertens, M. Álvarez, and J. Tamayo, Appl. Phys. Lett. 87, 1 (2005).

${ }^{19}$ P. Lu, H. P. Lee, C. Lu, and S. J. O'Shea, Phys. Rev. B 72, 085405 (2005).

${ }^{20}$ J. W. Yi, W. Y. Shih, and W.-H. Shih, J. Appl. Phys. 91, 1680 (2002).

${ }^{21}$ N. G. Stephen, J. Sound Vib. 131, 345 (1989).

${ }^{22}$ X. Yao, M. Jericho, D. Pink, and T. Beveridge, J. Bacteriol. 181, 6865 (1999). 